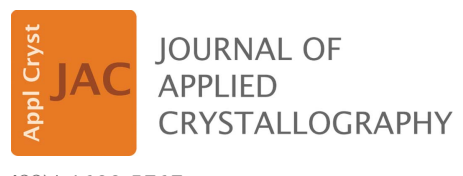

ISSN 1600-5767

Keywords: computer programs; diffraction simulation

\section{SingleCrystal 4: real-time multi-phase diffraction simulation}

\author{
David C. Palmer*
}

CrystalMaker Software Ltd, Centre for Innovation, Oxford University Begbroke Science Park, Woodstock Road, Begbroke, Oxfordshire OX5 1PF, UK. *Correspondence e-mail: david@crystalmaker.com

CrystalMaker Software Ltd has announced the release of a new version of their SingleCrystal program: a tool for understanding the diffraction properties of crystalline materials.

Designed for physicists, chemists, material scientists and geologists, SingleCrystal 4 can simulate the results of laboratory diffraction experiments - in real time, and from the comfort of the user's laptop. Observed electron diffraction images can also be analysed and their crystal orientations calculated, saving hours of painstaking measurement and analysis.

SingleCrystal 4 features a brand new design: the source code was completely rewritten to take advantage of the latest 64-bit multi-core operating systems with high-resolution graphics and multi-touch control. A new interface delivers a streamlined easy-to-use workflow and offers real-time adjustment of experimental and sample parameters.

A key feature of SingleCrystal 4 is the ability to simulate multi-phase diffraction - to replicate real-world effects such as 'twinning', topotactic relationships, crystal coatings and impurities - as well as showing 'Kikuchi lines' in transmission electron microscope patterns and 'powder rings' from nanocrystalline samples.

Whilst SingleCrystal 4 can be used as a standalone program - to create new simulations from scratch, or import crystal data files from other programs - it also works seamlessly with CrystalMaker X, allowing users to edit crystal structures at the atomic level (in CrystalMaker) and immediately see how this affects the diffraction properties: an ideal teaching tool.

SingleCrystal 4 is available as true native 64-bit Mac and Windows applications. The Mac version works with Catalina, offering Retina graphics, 'Dark Mode', multi-touch, haptic feedback and a dedicated Touch Bar interface for MacBook Pro. The Windows version is designed to run on the latest generation of Windows PCs and tablets, with highDPI displays and touch-sensitive control.

The software is sold directly by CrystalMaker Software Ltd (http://crystalmaker.com/ singlecrystal/). Multi-user, education and student licencing options are available.

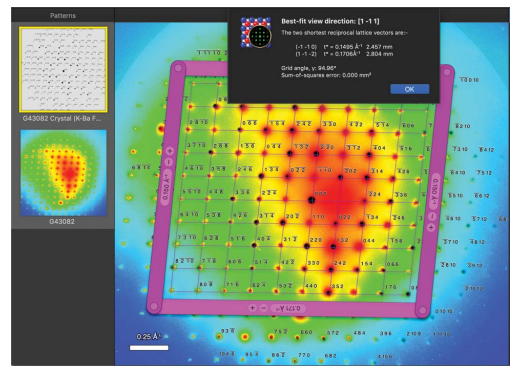

(C) 2020 International Union of Crystallography 